\title{
Review
}

\section{Care of the patient with invasive meningococcal disease by emergency medical service clinicians: a scoping review protocol}

James Pearce BN, MAdvPrac, BHSc, FPA is a PhD candidate and lecturer ${ }^{1}$, and a paramedic²; Micah DJ Peters BHSc, MA(Q), PhD is Research Fellow3; Nikki May BCultTour, MLiblnfMngt is a reference librarian4; Helen Marshall MBBS, MD, MPH, DCH is Professor in Vaccinology ${ }^{5}$, Deputy Director ${ }^{6}$ and Medical Director ${ }^{7}$; Cindy Hein ASM, BHSc, DipAppSc, PhD, FPA is Research Fellow ${ }^{1}$ and an extended care paramedic ${ }^{2}$; Hugh Grantham ASM, MBBS, FRACGP is Adjunct Professor ${ }^{8}$ and Senior Clinical Advisor ${ }^{2}$

\section{Affiliations:}

${ }^{1}$ Flinders University, Adelaide, South Australia

2SA Ambulance Service

${ }^{3}$ Rosemary Bryant AO Research Centre, School of Nursing and Midwifery, University of South Australia

${ }^{4}$ SA Health Library Service

${ }^{5}$ School of Medicine, The University of Adelaide, South Australia

${ }^{6}$ Robinson Research Institute, The University of Adelaide, South Australia

${ }^{7}$ Vaccinology and Immunology Research Trials Unit, Discipline of Paediatrics, The Women's and Children's Hospital, Adelaide, South Australia

${ }^{8}$ Curtin University, Perth, Western Australia

https://doi.org/10.33151/ajp.16.686

\section{Abstract}

\section{Background}

Invasive meningococcal disease (IMD) is a rare but potentially fatal disease. It can rapidly progress within minutes to hours and carries a significant burden of disease worldwide. The role of emergency medical service (EMS) clinicians in providing early care for the patient with IMD is important, but the literature regarding the pre-hospital phase of care is sparse.

\section{Aim}

The aim of this scoping review is to systematically map the literature to identify the scope, depth and key concepts in the evidence, and to identify gaps in knowledge regarding the care of the patient with IMD by EMS clinicians.

\section{Methods}

A comprehensive electronic search strategy will be used to locate sources of evidence that fit the inclusion criteria of the study. Data will be extracted, charted and reported in accordance with current best practice for scoping reviews.

\section{Discussion}

This protocol provides the framework for the first scoping review to identify the scope, depth and key concepts in the evidence, and to identify gaps in knowledge regarding the care of the patient with IMD by EMS clinicians. The review report will present the current state of knowledge, inform clinical practice guideline development and identify gaps in the evidence requiring further research.

\section{Keywords:}

ambulance; emergency medical services; meningitis; meningococcal disease; scoping review; sepsis

Corresponding Author: James Pearce, james.pearce@flinders.edu.au 


\section{Background}

\section{Rationale \\ Meningococcal disease overview}

Invasive meningococcal disease (IMD), caused by Neisseria meningitidis, is one of the most rapidly progressive infectious diseases. Despite advances in clinical management, it still carries a significant burden of disease worldwide (1). Although it is relatively rare in developed countries, it remains a significant public health concern due to its sudden onset, unpredictable nature and rapid clinical course. Due to its fulminant nature it is important that early identification and appropriate management is provided by emergency medical service (EMS) clinicians to reduce morbidity and mortality, and to ensure the best patient outcomes.

Invasive meningococcal disease has comparatively high mortality rates of about $10 \%$, and $20-50 \%$ for patients who present with shock (2). Patients who do survive often face debilitating sequelae such as amputation of digits and limbs, skin scarring, cognitive impairment and seizures $(3,4)$. A particularly troubling feature is that the most vulnerable population are children less than 1 year of age (5).

The meningococcus is often transiently and asymptomatically carried in the nasopharynx, and can be isolated in about 10\% of the general population at any time (6), although this varies widely depending on geographic and epidemiological setting (7). Sporadically and for reasons not completely understood, the meningococcus can invade the body where it can cause a spectrum of disease, from mild transient meningococcemia through to meningitis and fulminant meningococcal septicaemia. These severe forms of the disease are referred to as 'invasive meningococcal disease'.

The only known reservoir for the meningococcus is humans and it is spread by droplet transmission or direct contact with respiratory secretions from an infected host (5). The meningococcus serotypes responsible for causing the majority of disease are the $A, B, C, W, X$ and $Y$ strains (8).

Invasive meningococcal disease is predominantly comprised of two distinct presentations: meningococcal meningitis and meningococcal septicaemia. Often the two forms of the disease are not well distinguished in the literature and the term 'meningitis' is incorrectly used interchangeably for both (9). Meningococcal meningitis is infection of meninges (the lining of the brain and spinal cord) by N. meningitidis and carries a risk of death of approximately $5 \%$ (10). Meningococcal sepsis is a more serious form of the disease and can present with profound shock and cardiovascular collapse. It carries a risk of death of between $20-50 \%$ (2). Rarely, meningococcal disease may present in other forms such as conjunctivitis (11) and pericarditis (12).

\section{Meningococcal septicaemia}

At the most severe end of the IMD spectrum is fulminant meningococcal septicaemia, which can be fatal in less than 24 hours (13). Meningococcal septicaemia is a form of sepsis caused by the meningococcus. Sepsis is a clinical syndrome characterised by life-threatening organ dysfunction caused by a deregulated host response to infection (14). Seminal research by Rivers et al highlighted that early recognition and aggressive management of sepsis significantly improves outcomes (15). In patients with hypotension secondary to septic shock it has been demonstrated that each hour of delay in administration of antimicrobial agents increases patient mortality by $7.6 \%$ (16).

In a Scottish study, more than $80 \%$ of patients with sepsis arrived at the emergency department via EMS (17).

Recognition of sepsis by EMS clinicians is generally poor (18), with a Dutch study highlighting that EMS clinicians recognised sepsis in less than $15 \%$ of cases (19). The pre-hospital link in the chain of care is time where critical interventions could be initiated to improve patient outcomes. There remain many gaps in the knowledge in this area and further research is warranted (20).

\section{Issues with identification of IMD}

Invasive meningococcal disease has a variable time course and diverse clinical spectrum (21). This is especially evident in the first few hours when it can be difficult for clinicians to differentiate IMD from milder, self-limiting illnesses such as viral respiratory infections (22). Non-specific clinical features such as fever are common, and the characteristic petechial or haemorrhagic rash often appears late if at all. Because of its rarity most clinicians will have limited experience and expertise with the disease (22). Research undertaken in primary care (ie. general practice) confirms that recognition of IMD is difficult in that setting (23). It is reasonable to assume that EMS clinicians face similar challenges in their pre-hospital field, however literature in this area is sparse.

\section{Issues with management of IMD}

Mortality and morbidity in patients with IMD depends on many variables including the clinical manifestation (eg. septicaemia and/or meningitis), characteristics of the organism (eg. strain and virulence), patient characteristics (eg. age and immune status) and clinical management. Of these variables, the health care team can only influence one aspect; clinical management (24).

Guidelines state that for meningococcal septicaemia, prompt initiation of antibiotic therapy is recommended in the prehospital field (25). The evidence base for this recommendation is somewhat conflicting and inconclusive, with earlier studies finding a reduced mortality rate when pre-hospital parenteral antibiotics were administered in the primary care setting for IMD (26-28), and later studies finding an increased mortality 
rate (29-31). Due to the difficult nature of studying a rare disease and the ethical implications of withholding antibiotics in suspected cases of IMD, no randomised controlled trials exist on this topic. The current evidence is from the general practice setting (not EMS) and is based on retrospective observational data with its inherent biases (including confounding by severity and indication). Significant clinical heterogeneity exists due to the variable clinical presentation, clinical course and evolution of IMD.

Much of the literature regarding the pre-hospital management of IMD centres around the administration of parenteral antibiotics. The evidence for other pre-hospital interventions for IMD including oxygen therapy, fluid therapy, vasopressors, adjunctive therapies and hospital pre-alert notifications is scarce.

\section{Aim}

The aim of this scoping review is to systematically map the literature to identify the scope, depth and key concepts in the evidence, and to identify gaps in knowledge regarding the care of the patient with IMD by EMS clinicians. The review question is: What is the state of knowledge regarding the care of the patient with IMD by EMS clinicians in the international literature?

\section{Methods}

Scoping reviews are a methodologically rigorous approach to evidence synthesis (32), and can be used to identify the nature and extent of the research conducted on a topic (33). They can be used to map the literature, collate the evidence on a topic and summarise the characteristics and findings of the studies. They are particularly useful as a review methodology where there is a body of literature which has not yet been comprehensively reviewed (34) such as the present review topic which seeks to explore the characteristics and overall parameters of what has been studied in this field. The first framework for conducting scoping reviews was published by Arksey and O'Malley in 2015 (35) and has been extended and refined several times since (36-39).

This review will follow the most recently published methodology for scoping reviews, the Preferred Reporting Items for Systematic Reviews and Meta-Analyses extension for Scoping Reviews (PRISMA-ScR) (39), which is consistent with the methods described by Peters et al (38) and the Joanna Briggs Institute (40). This protocol has been prepared in line with the PRISMA Protocols (PRISMA-P) checklist. The protocol for this scoping review was also registered prospectively with the Open Science Framework (41) on 25 September 2018 (https://osf. io/ubd7w/), and was updated on 7 December 2018 following several minor updates (https://osf.io/z639u/).
A preliminary search of PROSPERO, Open Science Framework, Epistemonikos, PubMed, JBI Database of Systematic Reviews and Implementation Reports, and the Cochrane Database of Systematic Reviews did not locate any existing or underway reviews that address the objective of this review. Two published systematic reviews investigating the effectiveness of pre-admission antibiotics for IMD were located but do not include any EMS data $(24,42)$. Three published systematic reviews investigating EMS identification or management of sepsis were located but do not explicitly discuss IMD $(18,20,43)$.

The preliminary search found a number of publications of varying quality reporting on aspects of the care of the patient with IMD by EMS clinicians which will likely be included in the scoping review report (44-50).

\section{Inclusion criteria \\ Population}

This scoping review will include sources that focus on the care of patients (either actual or potential/theoretical) of any age, gender, ethnicity, pregnancy status or co-morbidities with suspected or confirmed IMD. This includes patients with meningococcal septicaemia, meningococcal meningitis and mixed clinical presentations, as defined by the individual sources.

Sources that report on patients with suspected or confirmed sepsis/septicaemia from causes other than IMD will not be included. This is because IMD has a clinical course that is distinct from other causes of sepsis (such as sepsis from a urinary or pulmonary origin).

\section{Concept}

This scoping review will include sources that report on any aspect of the care of IMD by EMS clinicians, which may broadly be categorised as either identification or management. Identification may include but is not limited to: factors regarding the identification, assessment, recognition, clinical judgement or provisional diagnosis relating to IMD, such as screening tools/items, clinical history, risk factors or the presence or absence of certain signs and symptoms such as a rash. Management may include but is not limited to: clinical decision making, oxygen therapy, fluid therapy, vasopressors, the administration of antibiotics, adjunct therapies (eg. paracetamol or steroids), hospital pre-alert notifications and disposition choices.

\section{Context}

The context of this scoping review is the emergency medical service, which may also be known as an ambulance service or paramedic service, and will include sources that report on EMS clinicians including but not limited to paramedics, nurses, medical practitioners and the various levels of ambulance 
technician (eg. first responders, emergency medical technicians or ambulance officers) delivering patient care in EMS systems. EMS systems which operate either FrancoGerman, Anglo-American, Basic Life Support or Advanced Life Support structures as defined by Al-Shaqsi (51) will be included. Literature that reports on in-hospital care, including emergency department or intensive care/therapy unit care, or non-EMS pre-hospital care (eg. general practice) will not be included in this scoping review.

\section{Types of evidence sources}

Due to the scarcity of literature on the review topic objective, all types of sources of evidence, including text and opinion literature (eg. expert opinion, comments, reviews and narratives) will be included as these may constitute the best available evidence (52).

Unpublished (grey) literature from the sources listed in the search strategy will be considered if relevant to the research question. Textbooks, social media posts, blogs and websites will not be included in this review.

EMS clinical practice guidelines (CPGs) pertaining to IMD will not be included in this review as they will be reviewed and comprehensively analysed using the Appraisal of Guidelines for Research and Evaluation AGREE II instrument (53) (a tool to assess the methodological quality of CPGs) in separate future publications.

\section{Search strategy}

For published literature, a three-step search strategy as described by Aromataris and Riitano (54) will be utilised. This will be an iterative process, as further literature and key words are found. First, an initial limited search of the Ovid MEDLINE and CINAHL databases will be undertaken. An analysis of the text words contained in the title and abstract of relevant literature and the index terms used to describe the article will be carried out. Second, a search using all identified keywords and index terms will be undertaken across all included databases. All potentially relevant citations will be retrieved in full text for assessment against the inclusion criteria before inclusion. Finally, the reference lists of all included literature will be screened for additional literature.

Searches will be restricted by publication date, commencing from 1992. This was the year of several publications regarding the use of antibiotic therapy for IMD in primary care settings $(23,26-28,55)$. It is highly unlikely that there will be any relevant published literature earlier than this date, as the first EMS CPGs for IMD were not released until 2000 (44). The search strategy will be developed in collaboration with a research librarian with expertise in systematic literature searching, as this has been found to increase the quality of the search strategy (56). A draft search strategy using medical sub-headings (MeSH) and free text terms for MEDLINE can be found in Table 1. The search strategy will be refined and adapted as necessary for the other databases and searches, and the final search strategies for all databases will be published in the review report.

Table 1. Search strategy for MEDLINE (Ovid interface)

\begin{tabular}{|c|c|}
\hline$\#$ & Searches \\
\hline 1 & $\begin{array}{l}\text { meningococcal infections/ or meningitis, } \\
\text { meningococcal/ or exp Neisseria meningitidis/ }\end{array}$ \\
\hline 2 & (meningococc ${ }^{*}$ or meningitis or neisseria).tw,kf. \\
\hline 3 & or/1-2 \\
\hline 4 & exp Emergency Medical Services/ \\
\hline 5 & Emergency Medical Technicians/ \\
\hline 6 & $\begin{array}{l}\text { (emergency medical service* or EMS or emergency } \\
\text { medical technician* or EMT or emergency service* } \\
\text { or prehospital or pre-hospital or preclinical or } \\
\text { pre-clinical or ambulance* or out-of-hospital or } \\
\text { paramedic }{ }^{*} \text { or first responder }{ }^{*} \text { or HEMS or field } \\
\text { triage).tw,kf. }\end{array}$ \\
\hline 7 & or/4-6 \\
\hline 8 & and $/ 3,7$ \\
\hline
\end{tabular}

\section{Information sources}

For published literature the following databases will be searched: MEDLINE (OVID interface), Embase and Emcare (OVID interface), CINAHL Complete (EBSCO), Scopus and Web of Science. To ensure comprehensiveness a search of Google Scholar will also be carried out $(57,58)$. The Google Scholar search will be limited to the first 200 results, ordered by relevance.

Including grey literature in the search strategy helps minimise the risk of publication bias (59). A search for theses will be carried out in ProQuest Dissertations and Theses, Open Thesis, Trove and the Networked Library of Theses and Dissertations. Clinical trial registries should also be included in systematic searches to identify completed and ongoing clinical studies (60). Included databases will be: ClinicalTrials.gov, Central Register of Controlled Trials, WHO International Clinical Trials Registry Platform and ISRCTN.

A Google search will also be undertaken using the advanced search function. In order to avoid personalisation of search results, searches will be performed after a fresh install of a new browser, limited to the first 200 results, ordered by relevance. A limitation of electronic database searches is that they may not reveal all possible literature on a topic (61). In order to ensure that all possible literature will be included in the review a hand search (limited to the past 10 years) will also be undertaken in the following peer-reviewed journals of high relevance to EMS:

- Australasian Journal of Paramedicine (formerly the Journal of Emergency Primary Health Care)

- British Paramedic Journal 
- International Paramedic Practice

- Irish Journal of Paramedicine

- Journal of Paramedic Practice

- Prehospital and Disaster Medicine

- Prehospital Emergency Care.

The corresponding authors of included sources will also contacted, the reference lists of all included sources will be scanned, and social media will be used in an attempt to identify any potential additional sources.

Selection of sources of evidence for inclusion will be determined by two independent reviewers in accordance with the above inclusion and exclusion criteria. If any disagreement arises between the two reviewers, it will be resolved through consensus or discussion with the broader review group.

\section{Data extraction/charting the evidence}

An initial data charting table has been developed to extract information relevant to the review question. The table will be piloted on a subset of relevant sources of evidence and refined further if necessary prior to extracting data from all included sources. Due to the iterative nature of scoping reviews it may be necessary for further refinements during the process of data extraction and these will be noted in the scoping review report. To ensure feasibility of the review, one reviewer (JP) will extract all the data and the results of each source will be verified by a second reviewer. A formal quality appraisal of included literature will not be undertaken in this review. This is consistent with the literature regarding the conduct of scoping reviews $(38-40)$.

\section{Presenting the results}

It is expected that there will be considerable variation in the methodological approach adopted across the literature including the details of included evidence sources and the outcomes reported. Data and results will be presented in tabular form with accompanying narrative descriptions. Further diagrammatic representation of the results may also be presented following consideration of the data by the review group.

\section{Discussion}

The authors of this scoping review believe it to be the first attempt to systematically map the literature to identify the scope, depth, key concepts and gaps in knowledge regarding the care of the patient with IMD by EMS clinicians. The scoping review method was chosen, as it is ideal in situations where scarcity of literature is likely. The review report will present the current state of knowledge, inform CPG development and identify gaps in the evidence requiring further research.

\section{Acknowledgements}

The review group would like to acknowledge the assistance of the secondary reviewer, Mr Robin Pap.

\section{Conflict of interest}

Micah Peters declares that he is a co-author of the PRISMA Extension for Scoping Reviews paper, and that he is the Chair of the Joanna Briggs Institute Methodology Group for Scoping Reviews. All other authors declare no known conflicts of interest.

\section{Funding}

James Pearce is financially supported by a National Health and Medical Research Council PhD scholarship through the Prehospital Emergency Care - Australia New Zealand Centre for Research Excellence (ID: 1116453), and receives an Australian Government Research Training Program Scholarship. Funding had no influence on this protocol.

\section{References}

1. Pace D, Pollard AJ. Meningococcal disease: clinical presentation and sequelae. Vaccine 2012;30(Suppl 2):B3-9.

2. Booy R, Habibi P, Nadel S, et al. Reduction in case fatality rate from meningococcal disease associated with improved healthcare delivery. Arch Dis Child 2001;85:386-90.

3. Wang B, Clarke M, Thomas N, Howell S, Afzali HHA, Marshall $H$. The clinical burden and predictors of sequelae following invasive meningococcal disease in australian children. Pediatr Infect Dis J 2014;33:316-8.

4. Strifler L, Morris SK, Dang V, et al. The health burden of invasive meningococcal disease: a systematic review. J Pediatric Infect Dis Soc 2016;5:417-30.

5. Australian Technical Advisory Group on Immunisation (ATAGI). The Australian Immunisation Handbook. 10th edn. The Australian Immunisation Handbook. Canberra: Australian Government Department of Health; 2017.

6. Trotter CL, Maiden MCJ. Carriage and transmission of Neisseria meningitidis. In: Feavers I, Pollard AJ, Sadarangani M, editors. Handbook of Meningococcal Disease Management. Switzerland: Springer; 2016. p. 1523.

7. Wertheim HFL, Horby P, Woodall JP. Atlas of Human Infectious Diseases. Oxford: Wiley-Blackwell; 2012.

8. Trotter C, Ramsay M, Harrison L. Introduction and epidemiology of meningococcal disease. In: Feavers I, editor. Handbook of Meningococcal Disease Management. Switzerland: Springer; 2016. p. 1-14. 


\section{References (continued)}

9. Hodgetts TJ, Brett A, Castle N. The early management of meningococcal disease. Emerg Med J 1998;15:72-6.

10. Brandtzaeg P. Pathogenesis and pathophysiology of invasive meningococcal disease. In: Frosch M, Maiden MCJ, editors. Handbook of Meningococcal Disease: Infection Biology, Vaccination, Clinical Management. Weinheim: Wiley; 2006. p. 427-80.

11. Dryden AW. Primary meningococcal conjunctivitis: an unusual case of transmission by saliva. Digit J Ophthalmol 2016;22:25-7.

12. Zeidan A, Tariq S, Faltas B, Urban M, McGrody K. A case of primary meningococcal pericarditis caused by Neisseria meningitidis serotype $Y$ with rapid evolution into cardiac tamponade. J Gen Intern Med 2008;23:1532-5.

13. Communicable Diseases Network Australia. Invasive meningococcal disease: CDNA National Guidelines for Public Health Units. 2017.

14. Singer M, Deutschman CS, Seymour CW, et al. The third international consensus definitions for sepsis and septic shock (Sepsis-3). JAMA 2016;315:801-10.

15. Rivers E, Nguyen B, Havstad S, et al. Early goal-directed therapy in the treatment of severe sepsis and septic shock. N Engl J Med 2001;345:1368-77.

16. Kumar A, Roberts D, Wood KE, et al. Duration of hypotension before initiation of effective antimicrobial therapy is the critical determinant of survival in human septic shock. Crit Care Med 2006;34:1589-96.

17. Gray A, Ward K, Lees F, Dewar C, Dickie S, McGuffie C. The epidemiology of adults with severe sepsis and septic shock in Scottish emergency departments. Emerg Med J 2013;30:397-401.

18. Smyth MA, Brace-McDonnell SJ, Perkins GD. Identification of adults with sepsis in the prehospital environment: a systematic review. BMJ Open 2016;6:e011218.

19. van der Wekken LCW, Alam N, Holleman F, van Exter P, Kramer MHH, Nanayakkara PWB. Epidemiology of sepsis and its recognition by emergency medical services personnel in the Netherlands. Prehospital Emerg Care 2016;20:90-6.

20. Lane D, Ichelson RI, Drennan IR, Scales DC. Prehospital management and identification of sepsis by emergency medical services: a systematic review. Emerg Med J 2016;33:408-13.

21. Nadel S. Treatment of meningococcal disease. J Adolesc Heal 2016;59:S21-8.

22. Thompson MJ, Ninis N, Perera R, et al. Clinical recognition of meningococcal disease in children and adolescents. Lancet 2006;367:397-403.

23. Sørensen HT, Møller-Petersen J, Krarup HB, Pedersen $\mathrm{H}$, Hansen $\mathrm{H}$, Hamburger $\mathrm{H}$. Diagnostic problems with meningococcal disease in general practice. J Clin Epidemiol 1992;45:1289-93.

24. Hahné SJM, Charlett A, Purcell B, et al. Effectiveness of antibiotics given before admission in reducing mortality from meningococcal disease: systematic review. BMJ 2006:332:1299-303.

25. National Institute for Health and Clinical Excellence (NICE). Meningitis (bacterial) and meningococcal septicaemia in under 16s: recognition, diagnosis and management [Internet]. 2010 [cited 2018 May 13]. Available at: www.nice. org.uk/guidance/cg102

26. Cartwright K, Reilly S, White D, Stuart J. Early treatment with parenteral penicillin in meningococcal disease. BMJ 1992;305:143-7.

27. Gossain S, Constantine CE, Webberley JM. Early parenteral penicillin in meningococcal disease. BMJ 1992;305:523-4.

28. Strang JR, Pugh EJ. Meningococcal infections: reducing the case fatality rate by giving penicillin before admission to hospital. BMJ 1992;305:141-3.

29. Harnden A, Ninis N, Thompson M, et al. Parenteral penicillin for children with meningococcal disease before hospital admission: case-control study. BMJ 2006;332:1295-8.

30. Sørensen HT, Nielsen GL, Schønheyder HC, et al. Outcome of pre-hospital antibiotic treatment of meningococcal disease. J Clin Epidemiol 1998;51:717-21.

31. Nørgård B. Pre-hospital parenteral antibiotic treatment of meningococcal disease and case fatality: a Danish population-based cohort study. J Infect 2002;45:144-51.

32. Munn Z, Peters MDJ, Stern C, Tufanaru C, McArthur A, Aromataris E. Systematic review or scoping review? Guidance for authors when choosing between a systematic or scoping review approach. BMC Med Res Methodol 2018;18:143.

33. Grant MJ, Booth A. A typology of reviews: an analysis of 14 review types and associated methodologies. Heal Inf Libr J 2009;26:91-108.

34. Khalil H, Peters M, Godfrey CM, Mclnerney P, Soares CB, Parker D. An evidence-based approach to scoping reviews. Worldviews Evidence-Based Nurs 2016;13:118-23.

35. Arksey H, O'Malley L. Scoping studies: towards a methodological framework. Int J Soc Res Methodol 2005;8:19-32.

36. Levac D, Colquhoun H, O'Brien KK. Scoping studies: advancing the methodology. Implement Sci 2010;5:69.

37. Daudt HML, van Mossel C, Scott SJ. Enhancing the scoping study methodology: a large, inter-professional team's experience with Arksey and O'Malley's framework. BMC Med Res Methodol 2013;13:1.

38. Peters MDJ, Godfrey CM, Khalil $\mathrm{H}$, et al. Guidance for conducting systematic scoping reviews. Int J Evid Based Healthc 2015;13:141-6.

39. Tricco AC, Lillie E, Zarin W, et al. PRISMA Extension for Scoping Reviews (PRISMA-ScR): checklist and explanation. Ann Intern Med 2018;169:467.

40. Peters MDJ, Godfrey C, Mclnerney P, et al. Scoping reviews. In: Aromataris E, Munn Z, editors. Joanna Briggs Institute Reviewer's Manual. The Joanna Briggs Institute; 2017. Available at: https://reviewersmanual.joannabriggs. 


\section{References (continued)}

41. Open Science Framework. OSF Home. 2018 [cited 2018 Sep 24]. Available from: https://osf.io/dashboard

42. Sudarsanam TD, Rupali P, Tharyan P, Abraham OC, Thomas K. Pre-admission antibiotics for suspected cases of meningococcal disease. Cochrane Database Syst Rev 2017;(6).

43. Smyth M, Brace-McDonnell S, Perkins G. Impact of prehospital care on outcomes in sepsis: a systematic review. West J Emerg Med 2016;17:427-37.

44. Cooke ME. Prehospital paramedic administration of benzyl penicillin by paramedics in the UK. Australas J Paramed 2005;3:1-6.

45. Burgess $S$. Social and environmental influences affecting the risk of development of meningococcal disease: considerations for prehospital care. Australas J Paramed 2006:4:1-10.

46. Hodkinson M. Understanding the process and treatment of meningitis and meningococcal disease. J Paramed Pract 2013:5:258-63.

47. Glennie L. Meningococcal septicaemia: identification and management by paramedics. ibid. 2011;3:606-7.

48. Tippett $V$, Bonham R. Review of the evidence for prehospital administration of benzyl penicillin in meningococcal septicaemia - experience in Queensland. Australas J Paramed 2005;3:1-10.

49. Walker T. Pre-hospital paramedic administration of ceftriaxone for suspected meningococcal septicaemia in Victoria, Australia. ibid. 2005;3:1-8.

50. Valenti P. Pre-hospital assessment and treatment of meningococcal meningitis. J Paramed Pract 2014;6:460-4.

51. Al-Shaqsi S. Models of international emergency medical service (EMS) systems. Oman Med J 2010;25:320-3.

52. McArthur A, Klugárová J, Yan H, Florescu S. Innovations in the systematic review of text and opinion. Int J Evid Based Healthc 2015;13:188-95.

53. Brouwers MC, Kho ME, Browman GP, et al. AGREE II: Advancing guideline development, reporting and evaluation in health care. J Clin Epidemiol 2010;63:1308-11.

54. Aromataris E, Riitano D. Constructing a search strategy and searching for evidence. Am J Nurs 2014;114:49-56.

55. Cartwright K, Strang J, Gossain S, Begg N. Early treatment of meningococcal disease. BMJ 1992;305:774.

56. Rethlefsen ML, Farrell AM, Osterhaus LC, Brigham TJ. Librarian co-authors correlated with higher quality reported search strategies in general internal medicine systematic reviews. J Clin Epidemiol 2015;68:617-26.

57. Bramer WM, Rethlefsen ML, Kleijnen J, Franco OH. Optimal database combinations for literature searches in systematic reviews: a prospective exploratory study. Syst Rev 2017;6:245.

58. Haddaway NR, Collins AM, Coughlin D, Kirk S. The role of google scholar in evidence reviews and its applicability to grey literature searching. PLoS One 2015;10:1-17.

59. Aromataris $E$, Pearson $A$. The systematic review: an overview. Am J Nurs 2014;114:53-8.

60. Baudard M, Yavchitz A, Ravaud P, Perrodeau E, Boutron I. Impact of searching clinical trial registries in systematic reviews of pharmaceutical treatments: methodological systematic review and reanalysis of meta-analyses. BMJ 2017;356:j448.

61. Armstrong R, Jackson N, Doyle J, Waters E, Howes F. It's in your hands: the value of handsearching in conducting systematic reviews of public health interventions. J Public Health (Bangkok) 2005;27:388-91. 